\title{
REDUCTION OF INTERFERENCE IN OBSERVATIONS WITH THE WESTERBORK SYNTHESIS RADIO TELESCOPE
}

\author{
T. A. TH. SPOELSTRA \\ Netherlands Foundation for Radio Astronomy \\ P. O. Box 2, 7990 AA Dwingeloo, The Netherlands
}

\begin{abstract}
Radio astronomical observations are increasingly affected by man-made interference of various kinds. Sophisticated software tools have to be developed to cope with interference effects in the data. A calibration procedure is described in which the effects of different categories of errors are separated. If this separation can be performed for interference effects, proper filtering tools allow for adequate rejection of these effects.

Interference effects remaining in the data are erroneously interpreted as source structure by self-calibration techniques. This is due to the fact that self-calibration alone does not properly take into account this separation. Some results for the Westerbork Synthesis Radio Telescope are given.
\end{abstract}

\section{INTRODUCTION}

Man-made interference of various kinds has an increasingly negative impact on observational astronomy. Many, if not all, radio astronomical observing sites have experienced some types of effects degrading the observations. Those observatories which have not been affected too much yet, will be affected in the foreseeable future, while many of the sites with negligible effects at present will be under severe pressure in the coming years. Planning for present and future radio astronomical observations must take these serious problems into account. Furthermore, sophisticated software tools have to be developed to cope with interference effects in the data (e.g. Kahlmann and Spoelstra, 1987; Spoelstra and Kahlmann, 1988). By means of these tools, the errors in the maps after calibration should be brought within the desired tolerance, i.e. within the maximum acceptable spread in amplitude gain errors allowed to reach the desired dynamic range.

Finally, the relative merits of the options of (a) repeat of the observation, (b) hardware improvement, and (c) use of software tools, should be evaluated regarding reduction of interference influences.

\section{MODULAR CALIBRATION}

The tolerances one derives for the corrected data (and also from those for the correction parameters) depends strongly on the distribution of the remaining errors over the U-V plane. It seems that an instructive way to consider this is to 
think of the Fourier-transform of the U-V plane to the map as an operation, which for each map position produces the real component of the weighted sum of all measured vectors in the U-V plane. (This, of course, after proper reorientation of those vectors, as given by the Fourier-transform.)

The input vectors for this summation consist of two pairs: noise and signal vectors affected by imperfections. The latter may be considered as the proper signal vectors plus error vectors, defined by the signal vectors and the fractional errors in complex amplitude (in the case of the Westerbork Synthesis Radio Telescope, WSRT, $1^{\circ}$ of phase corresponds to approximately $2 \%$ for small angles). For the same reason the resulting map may be considered as the sum of three maps:

a) the ideal, noise-free map;

b) the noise map;

c) the error map.

Of the two unwanted components, the noise map has a predefined rms amplitude determined by integration time and system noise. Our main concern is therefore the error map, which can be considered as a component of the skybrightness-distribution times the primary beam reception pattern, convolved with an unknown complex function. This is, of course, only a first order consideration, because if the errors in amplitude and phase are dependent on the position of the source within the image, the unknown convolving function will be also. This can be considered as a combination of a series of error functions, e.g. for atmospheric effects, for unwanted interference, for telescope dependent effects, or for baseline dependent effects.

Calibration of the data means then, that this unknown convolving function should be made as small as possible. The aim is to make it smaller than the noise. Tolerances for calibration are properly reached when this has been achieved. When this cannot be done, this function must be made as small as possible with respect to the synthesized antenna pattern.

Taking into account these considerations, we assume that the optimum calibration quality is reached when the different "components" of the unknown convolving function can be controlled separately. If not, these components may affect each other and the function cannot be controlled properly: e.g. instrumental, interference, atmospheric and other effects will enter a source model in case of self-calibration causing corruption of the final results. This approach of "modular calibration" is applied on observations with the WSRT. Basically, the calibration is then done in three phases:

Phase 1) determine correction factors for external effects (i.e. originating outside the instrument), such as due to the atmosphere and interference;

Phase 2) determining telescope-based errors;

Phase 3) self-calibration.

Methods to remove atmospheric effects have been reviewed elsewhere (Spoelstra, 1987a, 1987b). The sequence of the different steps in the calibration process is not trivial. The sequence taken here implies an optimum reduction of mixing the different kinds of errors. It is obvious that the self-calibration phase should be done on data as much as possible free of any distorting effects: it should, therefore, be the last step in the calibration. And before any 
instrumental errors can be detected, the data should be clean of errors due to external effects. Therefore, the external effects have to be corrected first. However, in order to detect any atmospheric errors, the data should already be free of interference. We conclude, therefore, that filtering man-made interference effects out of the data should be the first step in the calibration.

\section{FILTERING INTERFERENCE}

Interference effects are noticeable as distortion of the data as a function of time, frequency, polarization and interferometer spacing (or baseline). In the case of single dish observation, the latter characteristic is not seen, of course. This distortion affects both the amplitude and phase of the signal (or the cosine and sine equivalents). Any method to remove interference should be based on the assumption that the characteristics of the astronomical radio source are not known. This condition reduces the applicability of sophisticated filtertechniques, which assume a priori knowledge of the source. Therefore, for WSRT observations, we applied as a first attempt a simple algorithm to remove interference, i.e. to delete the information in the time, frequency, polarization and baseline domain affected by interference.

The method used for the present work is as follows: The median, $\mathrm{m}$, of all sine and cosine data at a single baseline is determined. Then for an hour angle (or time) interval $\mathrm{dt}$ running with time the median, $\mathrm{m}_{\mathrm{dt}}$, is determined. Since an interfering signal is in principle much stronger than the astronomical signal, we assumed that data having an amplitude larger than $D=f\left(m_{d t}-d\right)$ (where $d$ is the minimum value of all the data at this baseline and $f$ is a numerical factor) are bad data due to interference. Let the cosine median for interval $\mathrm{dt}$ be $\mathrm{m}_{\mathrm{c}, \mathrm{dt}}$ and the median for all data at this baseline be $M_{c}$. Then the maximum amount $m_{c, d t}$ and $M_{c}$ are allowed to deviate from the median is $L_{c}=D\left(c_{a} / A_{a}\right)$, where $c_{a}$, is the average cosine value and $A_{a}$ is the average amplitude value over the whole observation. Analogously the sine data are checked. In this manner the data at all baselines are checked. For the present analysis we used $\mathrm{f}=2$ and $\mathrm{dt}=5$ minutes.

\section{SELF-CALIBRATION}

After having removed as much interference effect as possible, corrections for e.g. atmospheric effects can be applied. Existing atmospheric models cannot be used to correct for atmospheric effects at time scales less than the duration of the observation and with spatial structures less than the linear dimensions of the instrument beams. This limitation illustrates the limitations of many tools to correct the observations. After the first phase where correction factors for external effects are determined, residual effects may still be present. These might be removed by a correctional method which works on the data themselves, taking into account known characteristics of the problem and of the instrument, and a model of the image (which can be improved iteratively). In radio astronomy this technique is called self-calibration (Spoelstra, 1985). This method implies, however, that the astrometric accuracy is reduced; it is determined by the absolute phase of the shortest baseline instead of the longest. 
Self-calibration has proven to be a successful approach to achieve high quality (i.e. high dynamic range) images.

There exist several methods for self-calibration, of which the techniques developed at NRAO (especially intended for VLA data) and at the Netherlands Foundation for Radio Astronomy (based on observations with the WSRT) are the two typical types. The difference between the two methods lies in the use of "redundancy" information (i.e. output from many interferometers with the same baseline length, but involving different telescopes).

In the case of WSRT observations, this phase is divided into two steps: one to remove errors depending on the interferometer element (phase 2 - see above) by using information of redundant baselines, and secondly the proper selfcalibration phase (phase 3 - see above). The former of these steps is essential to avoid any instrumental effects in the self-calibration phase which cause instrumental artifacts in the final results. Furthermore, in this phase any residual error might be interpreted as an instrumental error connected to the individual interferometer elements. Thus, when the redundant data are used, these errors are taken into account as well.

\section{USING REDUNDANT DATA}

In "non-redundancy" methods, the observational data go through a "basic" self-calibration process. By comparing the observed data with the prediction of a source model, a set of antenna-based amplitude and phase corrections is computed per hour angle scan, which would bring the data into a better agreement with the model. From the corrected data a new model is made, which is the input for another cycle of calibration. The process is repeated until the solution becomes stable.

In the "redundancy" method, the use of redundant information offers an opportunity to correct instrumental errors and external errors separately, because for different interferometers with the same baseline, only instrumental errors cause differences in their outputs. The redundancy calibration first compares all spacings that carry redundant information with a reasonable defined signal to yield a weighted least-squares solution for all telescope errors. After corrections for instrumental errors have been applied, the data go through the "basic" self-calibration cycles. Since the instrumental errors have been corrected by using redundancy information, the data quality has been improved before the "basic" self-calibration. A much-improved first model can also be built from these data. This significantly decreases the residual error of the following "basic" self-calibration and improves the resulting image (Yang, 1987, 1988). The disadvantage of this method is increasing complexity of the receiver hardware to obtain redundancy information.

The important difference between these approaches is that in the "nonredundancy" method one has to deal with all errors in the self-calibration process, while in the "redundancy" method instrumental errors have already been solved before the self-calibration phase. 


\section{DISCUSSION}

Summarizing the previous sections, an ideal calibration scheme might be along the following steps:

1) "Filter" interference effects

2) Correct for known external effects (as atmospheric influences)

3) Correct for instrumental effects

4) Self-calibration. In order to illustrate this procedure, we calibrated a WSRT observation done at $325 \mathrm{MHz}$ with 1 polarization and a beam width of 2.5 $\mathrm{MHz}$ by using and comparing the results of the following procedures:
a) no calibration
b) only step 4
c) step $2-4$
d) step 2-4 and interactive deletion of bad data
e) step 1-4

The resulting maps are presented in Figures 1 - 5 .

The observation suffered strong interference with a complex dependence on interferometer baseline, hour angle and frequency. Before discussing the results, we should note that each individual cosine and sine value is stored in 2 bytes. Then if, during the Fourier transform, overflow of the data occurred, these data would be automatically deleted. Therefore, the resulting map is always cleaner than if the data are stored in 4 bytes per individual number and less overflow events occur. Due to the internal accuracy of the data and confusion effects, the noise in the final maps will always be larger than the noise which can be achieved theoretically. This theoretical noise is at present $1.2 \mathrm{mJy}$ for the chosen configuration. The peak flux of the brightest source in the map is $4.25 \mathrm{Jy}$ (not yet corrected for beam attenuation). During the calibration only one iteration step has been done in each of the five different procedures. The results are as follows:

Step a) no calibration: The noise in the final map is $8 \mathrm{mJy}$ and at a level of about $30 \mathrm{mJy}$ the map shows an interference pattern of stripes through the map.

Step b) self-calibration: The noise in the final map is $12 \mathrm{mJy}$ and at a level of about $40 \mathrm{mJy}$ the map shows an interference pattern. It should be noted that the interference pattern in step a shows mainly a real component, while in this case the major component is imaginary. The results might be improved if more iterations are done. However, this would imply that the interference effects would be included in the source model components causing artifacts which are not real.

Step c) correction for external effects (not interference) and instrumental effects plus self-calibration: The results are similar to those of step a, but the noise is $10 \mathrm{mJy}$ and the interference pattern is somewhat enhanced.

Step d) as step c, but interactively the bad data have been removed: The noise in the final map is $8 \mathrm{mJy}$ but the interference in this map is clearly below this level.

$\underline{\text { Step }}$ e) In step d the interactive deletion means that human interaction with 
the data functions is an "algorithm" to filter the interference. Since this may imply much subjective bias, a software filter might be more desirable to remove interference effects. The filter as suggested in the section on "Filtering Interference" was used as a first attempt. First the data passed this filter and then the data were calibrated as step c. The results show that the noise in the final map is about $20 \mathrm{mJy}$ and that the effects of interference in the map are below this level. The relatively high noise level indicates that the filter used is far from optimum and work has to be done to improve it. Another problem with the used filter is that the data reduction took several hours computing time (cputime): about two times the duration of the observation, which is far too much in practical applications.

\section{CONCLUSIONS}

Calibration observations suffering from unwanted interference effects can well be done reasonably well if one is able to separate the different correction phases for the different types of errors. Applying self-calibration only to the data turns out to give rather bad results. Work has to be done to improve any software-filtering method to remove interference effects; i.e. to reduce the amount of processing time and optimize the interference rejection mechanism (not too much data should be rejected and not too few).

\section{REFERENCES}

Kahlmann, H. C., Spoelstra, T. A. Th., 1987, Netherlands Foundation for Radio Astronomy, Internal Technical Report 184.

Spoelstra, T. A. Th., 1985, Astron. Astrophys., 148, 21.

Spoelstra, T. A. Th., 1988, Publ. Astron. Obs. Beograd (in press).

Spoelstra, T. A. Th., Kahlmann, H. C., 1988, Netherlands Foundation for Radio Astronomy, Internal Technical Report 185.

Yang, Yi-pei, 1987, Netherlands Foundation for Radio Astronomy, Internal Technical Report 182.

Yang, Yi-pei, 1988, Astr. Astrophhys. (in preparation). 

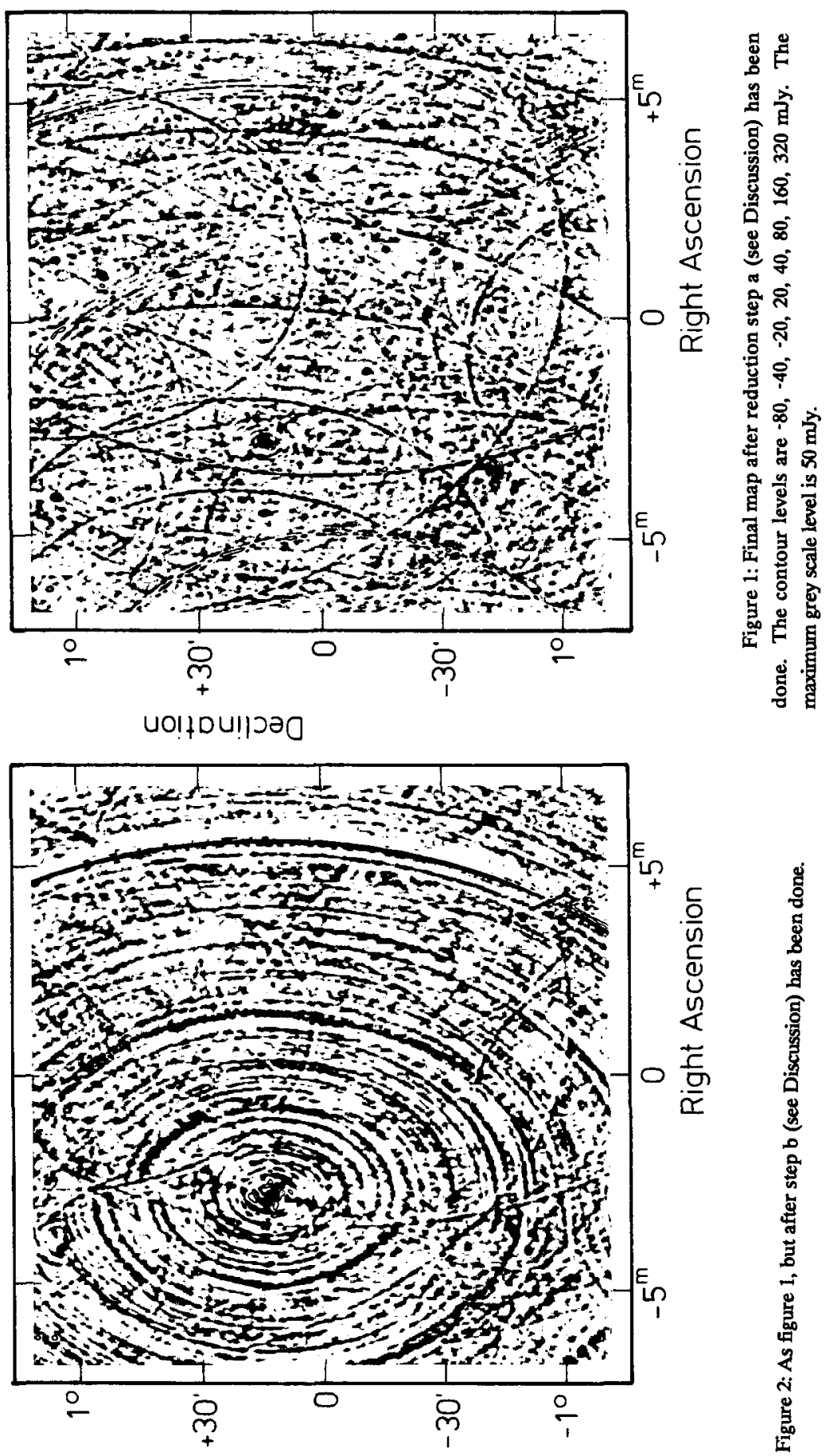

UO!łDU!jJaO 

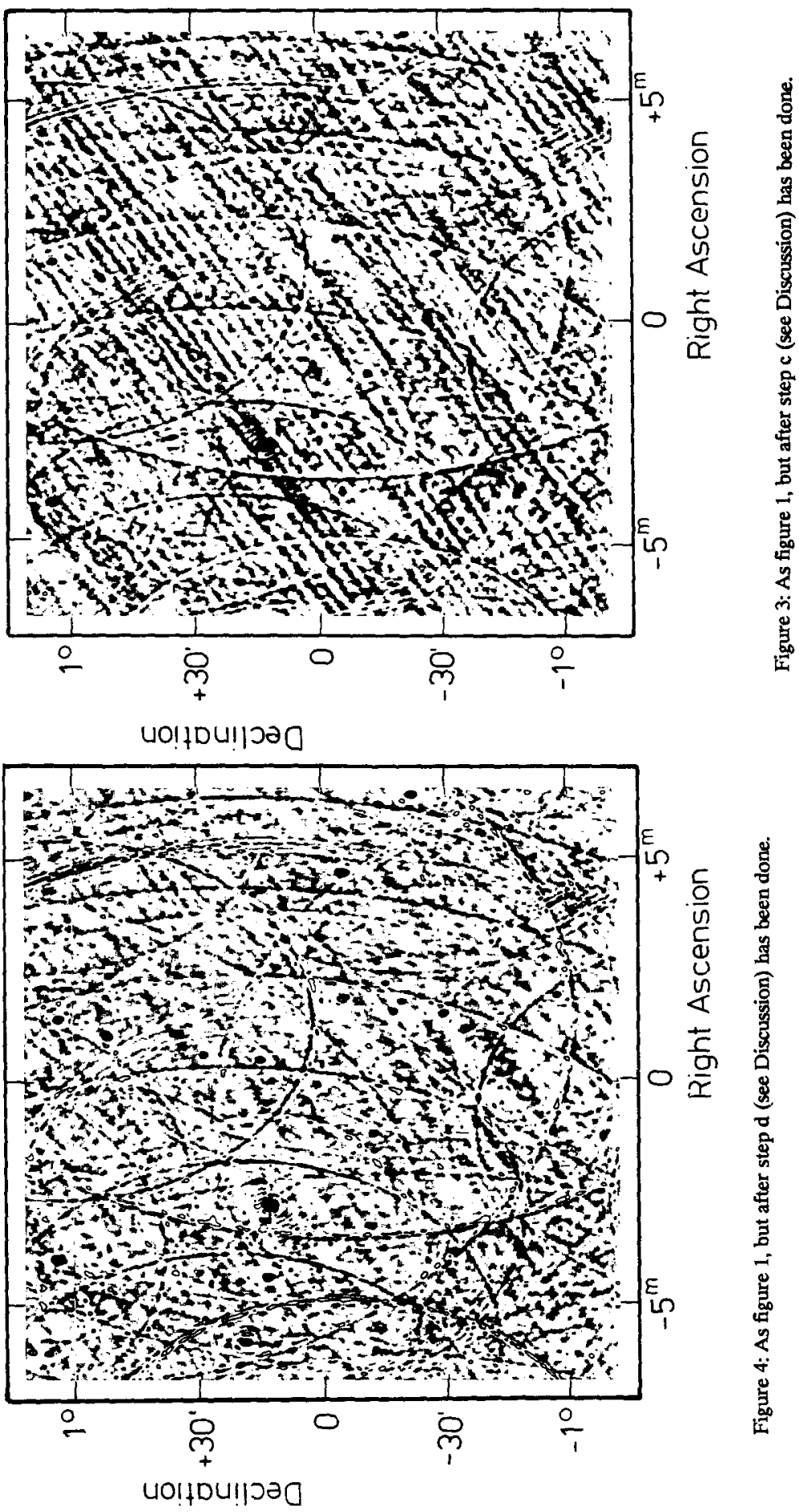

בं 


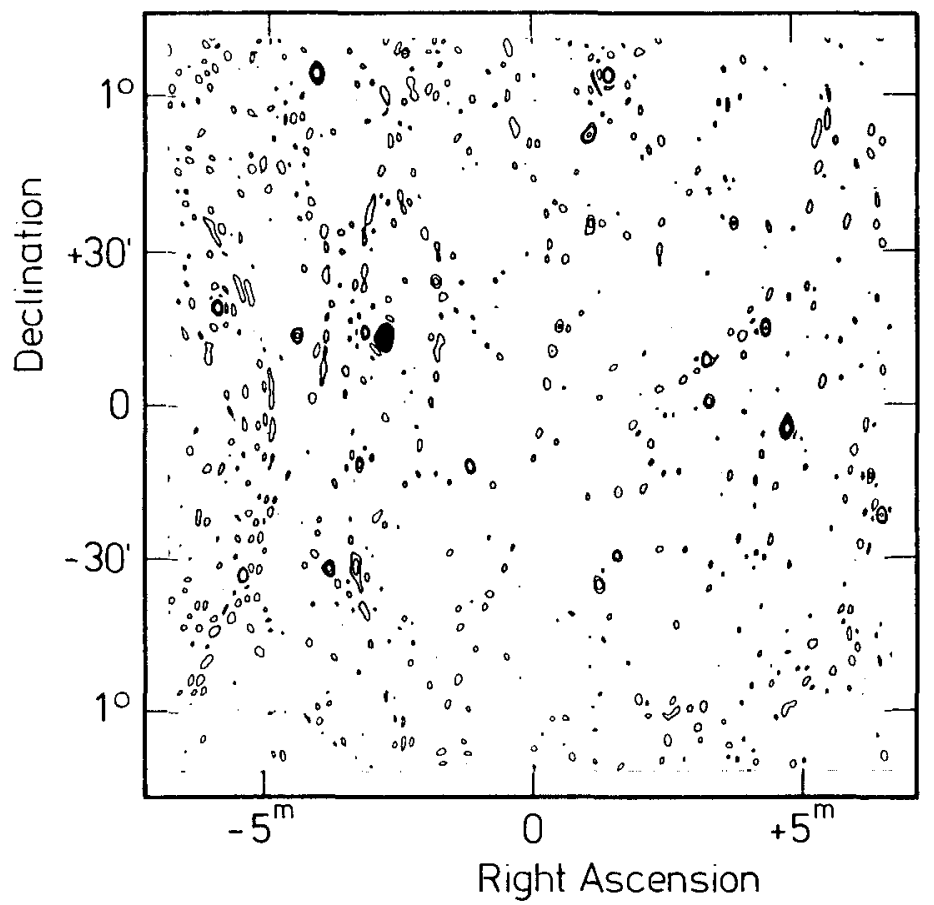

Figure 5: As figure 1, but after reduction step e (see Discussion) has been done. The maximum grey scale level is $4.3 \mathrm{Jy}$ (the peak flux in the map). 